\title{
Correlation between Severity of Coronary Artery Disease and Insulin Resistance in Diabetic Patients
}

\author{
Rehab I. Yaseen ${ }^{1}$, Mohammad Y. Beda*2, Fatma A. Ibrahim ${ }^{1}$
}

${ }^{1}$ Department of Cardiology, Faculty of Medicine, Menoufia University, Egypt

${ }^{2}$ Department of Cardiology, Dar El-Shefaa Hospital, Cairo, Egypt

*Corresponding author: Mohammad Y. Beda, Mobile (+20) 0109767 7504, E-mail: mohammed.yahya84@yahoo.com

\begin{abstract}
Background: Diabetes mellitus is a well-established risk factor for the development of coronary artery disease (CAD), which is the major cause of premature death in diabetic patients, both in type 1 or type 2 diabetes.

Objective: To examine the relationship between insulin resistance determined by fasting insulin level and complexity of coronary artery lesion in diabetic patients.

Patient and methods: This study enrolled 84 diabetic patients who were presented to the catheter Lab for elective coronary angiography in the period from October 2019 to April 2020. All patients were subjected to full history taking, thorough clinical examination, laboratory investigations (fasting insulin level, $\mathrm{HbA} 1 \mathrm{c}$ level, lipid profile and serum creatinine), 12-lead surface ECG, echocardiography examination and coronary angiography. The complexity of the coronary artery lesions was evaluated using Gensini score. Statistical tests were used to analyze the association between the measured fasting insulin levels and Gensini score.

Results: there was a statistically highly significant positive linear correlation between insulin level and Gensini score $(\mathrm{P}<0.05)$. The accuracy of for insulin level as a predictor for coronary artery disease equals $96,6 \%$ by $96.7 \%$ sensitivity and $75 \%$ specificity at cut off predictive value $<25.8 \mathrm{mIU} / \mathrm{L}$.

Conclusion: Fasting insulin level is a simple test for insulin resistance and significantly correlated with severe CAD so it may have a value as a predictor of severity of CAD and risk stratification of diabetic patients.
\end{abstract}

Keywords: Coronary artery disease, Diabetic patients, Insulin resistance.

\section{INTRODUCTION}

Coronary artery disease is the major cause of death worldwide, and more than $60 \%$ of the global burden of disease occurs in developing countries. GBD 2010 study showed that death from coronary artery disease increased by $87.8 \%$ between 1990 and 2010 , and a further rise by $50 \%$ is expected by 2030 (1). The prevalence of coronary artery disease in diabetes is not only high, but also characterized by increasing the severity and when the diabetic patients have the coronary artery disease, typically they have more severe disease, with a greater number of arteries involved ${ }^{(2)}$. Diabetes is a fast-growing health problem in Egypt with a significant impact on morbidity, mortality, and health care resources. Currently, the prevalence of type 2 diabetes (T2D) in Egypt is around $15.6 \%$ of all adults aged 20 to $79^{(3)}$.

Insulin resistance (IR) is a pathological condition in which cells fail to respond normally to insulin. IR known to be a pathogenic cause that can predict the occurrence of coronary artery disease ${ }^{(4)}$. The evolution of insulin resistance is unique in type 2 diabetes mellitus because it precedes the onset of diabetes and remains fairly constant throughout the disease process from the time of diagnosis and even after the conventional treatment for type 2 diabetes mellitus ${ }^{(5,6)}$.

Gensini score is a widely used angiographic scoring system for quantifying the severity of coronary artery disease, and was first described in
1975 by Goffedo G. Gensini. The Gensini score has been developed to characterize the complexity of coronary artery disease taking into consideration three main parameters for each coronary lesion: severity score, region multiplying factor and collateral adjustment factor ${ }^{(7,8)}$. Therefore aim of this work was to examine the relationship between insulin resistance determined by fasting insulin level and complexity of coronary artery lesion in diabetic patients.

\section{PATIENTS AND METHODS}

A total number of eighty four diabetic patients diagnosed according to the American Diabetes Association (ADA) were referred to elective coronary angiography at Dar El-Shefaa Hospital within the time period from October 2019 to April 2020, and patients with sepsis, pregnancy, acute or chronic renal failure, active bleeding, severe anemia, decompensated heart failure were excluded from the study.

All patients were subjected to history taking, full clinical examination, venous samples were withdrawn for fasting insulin level, glycated hemoglobin, serum creatinine and lipid profile. Electrocardiogram was done, and coronary angiography was recorded, and Gensini scores were calculated then patients were divided into four groups according to Gensini: Group (1): Normal coronary arteries (0 score), Group (2): Mild CAD (0-15 
scores), Group (3): Moderate CAD (16-30 scores) and Group (4): Severe CAD (31 -72 scores).

\section{Ethical consent:}

An approval of the study was obtained from Menoufia University Academic and Ethical Committee. Every patient signed an informed written consent for acceptance of sharing in the study. This work has been carried out in accordance with The Code of Ethics of the World Medical Association (Declaration of Helsinki) for studies involving humans.

\section{Statistical Analysis}

Data were collected, tabulated and statistically analyzed using an IBM compatible personal computer with Statistical Package for the Social Sciences (SPSS) version 23. Results were quoted as two-tailed probabilities. Significance of the obtained results was judged at the $5 \%$ level $(\mathrm{P} \leq 0.05)$.

\section{RESULTS}

The mean age of the study group was 58.76 \pm 9.22 years, 34 patients $(42.5 \%)$ were females, all patients were suffering from diabetes mellitus (all type

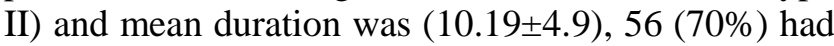
arterial hypertension, $45(56.2 \%)$ were suffering from dyslipidemia, $28(35 \%)$ patients had family history of coronary artery diseases and $26(32.5 \%)$ patients were smokers.

The mean left ventricular end diastolic diameter (LVEDD) was $(54.11 \pm 4.1) \mathrm{mm}$, mean end systolic diameter (LVESD) was $(35.14 \pm 4.2) \mathrm{mm}$ and mean ejection fraction (EF) was $(62.3 \pm 6.6 \%)$.

Mean insulin level in patients as regard their clinical presentation is shown in table (1).
Table (1): Insulin level in the studied groups as regard their clinical presentation

\begin{tabular}{|l|c|c|c|c|}
\hline & STEMI & NSTEMI & $\begin{array}{l}\text { Unstable } \\
\text { angina }\end{array}$ & $\begin{array}{c}\text { Stable } \\
\text { angina }\end{array}$ \\
\cline { 2 - 5 } & $\mathbf{N = 2}$ & $\mathbf{N}=\mathbf{1 6}$ & $\mathbf{N}=\mathbf{1 2}$ & $\mathbf{N = 5 4}$ \\
\hline $\begin{array}{l}\text { Insulin } \\
\text { level } \\
\text { (mIU/L) }\end{array}$ & $28.8 \pm 3.9$ & $22.1 \pm 4.9$ & $19.4 \pm 3.5$ & $19.1 \pm 4.7$ \\
\hline
\end{tabular}

Data are presented as mean \pm SD

Regarding hypertension, hypertensive patients had a highly significant insulin level than nonhypertensive. Moreover, patients with dyslipidemia had a significant insulin level than patients with normal lipid profile. In contrary insulin level in patients with family history of coronary artery diseases or smokers showed non-significant relation, as shown in table (2).

Table (2): Comparison between insulin level in the studied patients according to their risk factors

\begin{tabular}{|c|c|c|}
\hline Risk factors & $\begin{array}{c}\text { Test of } \\
\text { significance }\end{array}$ & P-value \\
\hline Hypertension & 5.801 & $<\mathbf{0 . 0 0 1} * *$ \\
\hline Dyslipidemia & 2.148 & $\mathbf{0 . 0 3 2}$ \\
\hline Family history & 0.429 & 0.668 \\
\hline Smoking & 0.503 & 0.615 \\
\hline
\end{tabular}

**: Highly significant difference

In the current study, there was no statistically significant difference between the studied groups as regard age and sex.

As regard risk factors; hypertension was more prevalent in patients with coronary artery disease than those with normal coronary. But there was no statistically significant difference between the studied groups as regard smoking, dyslipidemia and family history as shown in table (3).

Table (3): Comparison between the studied groups as regard demographic data and risk factors

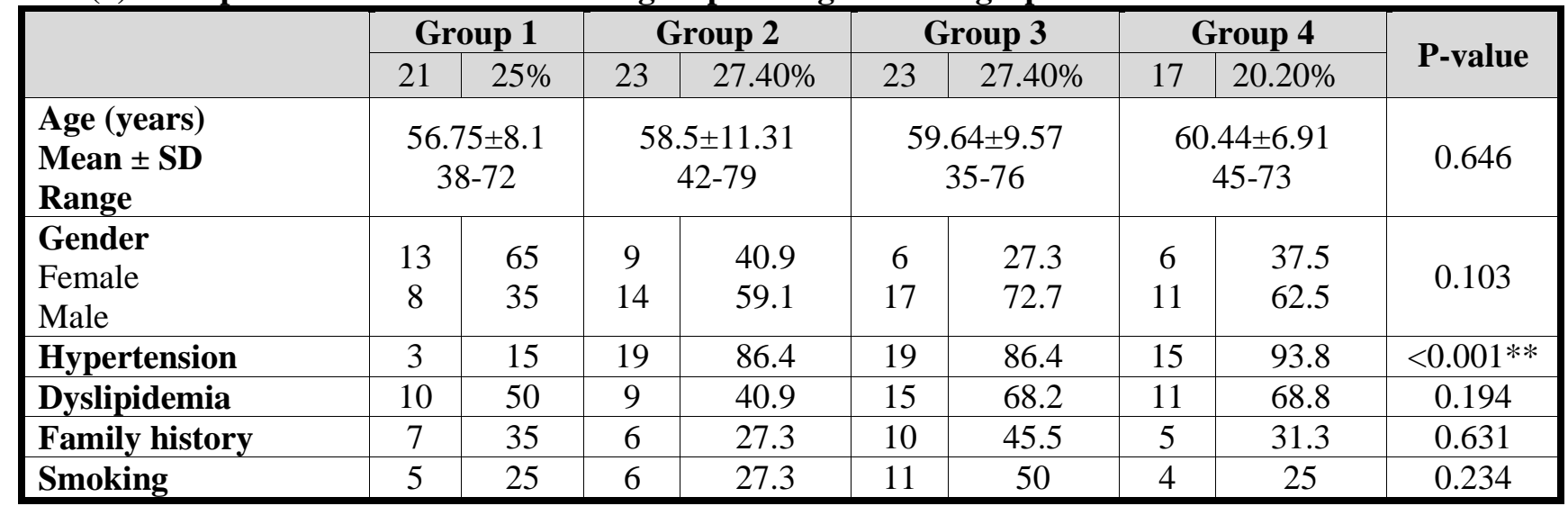

**: Highly significant difference

Also, by comparing conventional echocardiographic parameters in the studied groups, we found that LVESD was significantly larger in patients with severe coronary artery disease compared to other groups. But no significant difference was found between the studied groups as regard LVEDD. Moreover EF\% was significantly lower in patients with severe coronary artery disease than other groups as shown in table (4). 
Table (4): Comparison between the conventional echocardiographic parameters in the studied groups

\begin{tabular}{|c|c|c|c|c|c|c|c|c|c|}
\hline & \multicolumn{2}{|c|}{ Group 1} & \multicolumn{2}{|c|}{ Group 2} & \multicolumn{2}{|c|}{ Group 3} & \multicolumn{2}{|c|}{ Group 4} & \multirow{2}{*}{ P-value } \\
\hline & 21 & $25 \%$ & 23 & $27.40 \%$ & 23 & $27.40 \%$ & 17 & $20.20 \%$ & \\
\hline LEVDD & \multicolumn{2}{|c|}{$53.85 \pm 3.99$} & \multicolumn{2}{|c|}{$53.82 \pm 3.89$} & \multicolumn{2}{|c|}{$53.91 \pm 4.33$} & \multicolumn{2}{|c|}{$55.13 \pm 4.41$} & 0.754 \\
\hline \multirow{2}{*}{ LVESD } & \multicolumn{2}{|c|}{$\begin{array}{c}34.1 \pm 4.23 \\
29-45\end{array}$} & \multicolumn{2}{|c|}{$\begin{array}{c}34.68 \pm 3.41 \\
29-42\end{array}$} & & $\begin{array}{l}.91 \pm 3.24 \\
30-44\end{array}$ & \multicolumn{2}{|c|}{$38.75 \pm 4.59$} & \multirow{2}{*}{$<0.001^{* *}$} \\
\hline & & P1: 0.625 & P2: & P3: & & 4:0.507 P5 & $02 \mathrm{P}$ & .001 & \\
\hline \multirow{2}{*}{ EF\% } & & $5 \pm 5.1$ & & $6 \pm 6.13$ & & $.91 \pm 4.32$ & & $5 \pm 5.76$ & \multirow{2}{*}{$<0.001 * *$} \\
\hline & \multicolumn{2}{|c|}{ P1: 0.513} & P2: ( & $1 \quad$ P3:< & & $.978 \quad$ P5 & $01 \mathrm{~F}$ & 0.001 & \\
\hline
\end{tabular}

Data are presented as mean \pm SD and range

**: Highly significant difference

P1: between group 1and group $2 \quad$ P2: between group1 and group 3

P3: between group1 and group4

P4: between group2 and group3 P5: between group2 and group4

P6: between group 3 and group4

Regarding insulin level, it was significantly higher in patients with CAD than those with normal coronaries, moreover it was significantly higher in patients with severe CAD than those with mild and moderate $\mathrm{CAD}$, and in patients with moderate $\mathrm{CAD}$ than those with mild CAD.

Similarly, HbA1c was significantly higher in patients with CAD than those with normal coronaries, moreover it was significantly higher in patients with severe CAD than those with mild and significantly higher in patients with moderate CAD than those with mild CAD. As regard total cholesterol and they were significantly higher in patients with moderate and severe CAD than those with normal coronaries. In the contrary HDL level was significantly lower in patients with CAD than those with normal coronaries, moreover it was significantly lower in patients with severe CAD than those with mild and moderate CAD, and in patients with moderate CAD than those with mild CAD. Lastly regarding LDL level, it was significantly higher in patients with CAD than those with normal coronaries, as shown in table (5).

Table (5): Comparison between the laboratory data in the studied groups

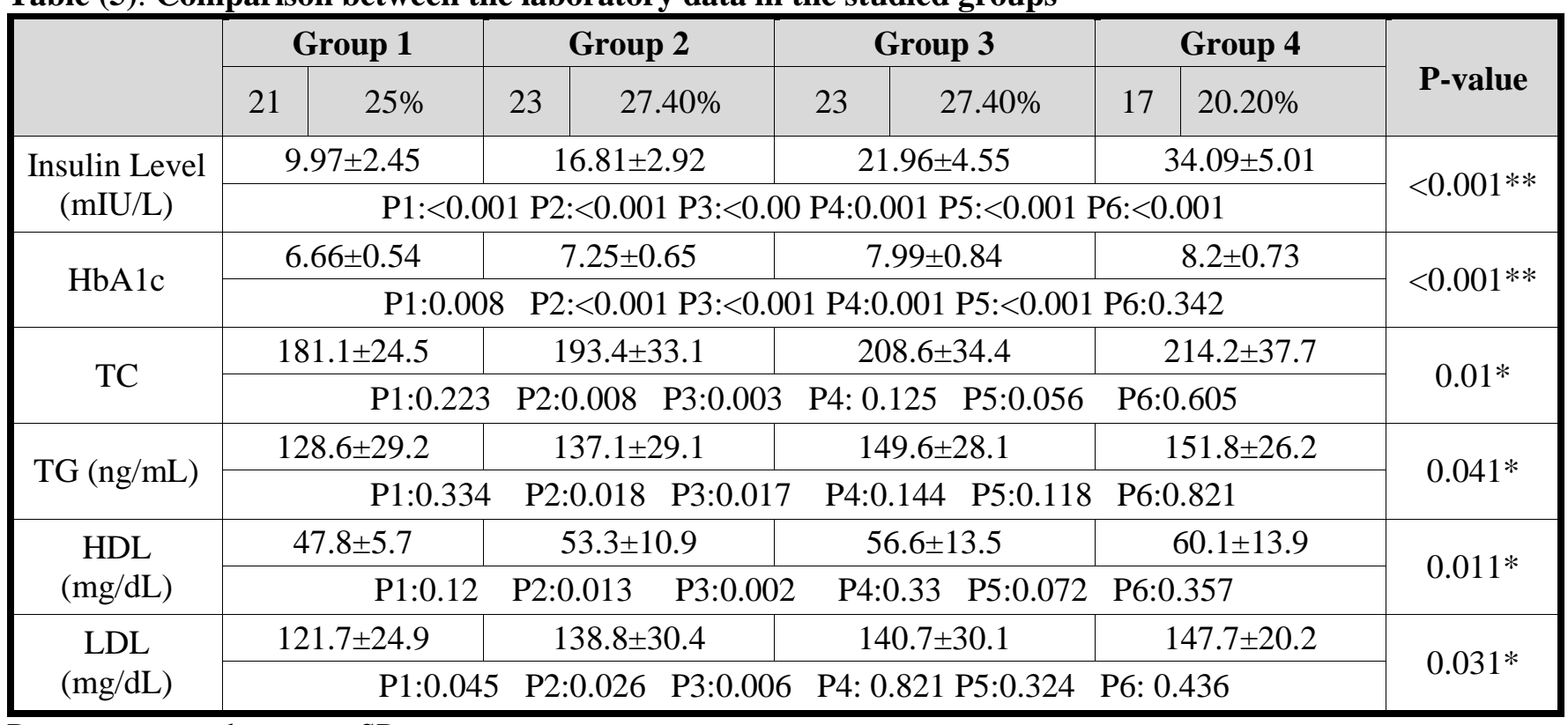

Data are presented as mean \pm SD

*: Significant difference **: Highly significant difference

P1: between group 1and group 2 P2: between group1 and group 3

P3: between group1 and group4

P4: between group2 and group3 P5: between group2 and group4

P6: between group 3 and group4 
Table (6) there was a statistically highly significant positive linear correlation between insulin level and Gensini Score. On the contrary there was a statistically highly significant negative linear correlation between insulin level and EF\%. But no significant correlation was found between fasting insulin level and duration of diabetes.

Table (6): Correlation between insulin level and other parameters in the studied patients

\begin{tabular}{|l|c|c|}
\hline \multirow{2}{*}{} & \multicolumn{2}{|c|}{ Insulin level } \\
\cline { 2 - 3 } $\begin{array}{l}\text { Duration of } \\
\text { DM (years) }\end{array}$ & -0.075 & 0.509 \\
\hline EF\% & -0.405 & $<0.001^{* *}$ \\
\hline Gensini score & 0.837 & $<0.001^{* *}$ \\
\hline
\end{tabular}

**: Highly significant difference

Table (7) revealed the results derived from the ROC curve showed that the accuracy of insulin level as a predictor for coronary artery disease $96.6 \%$ by $96.7 \%$ sensitivity and $75 \%$ specificity, at a cut off predictive value $\geq 25.8$.

Table (7): ROC curve for insulin level as a predictor for coronary artery disease in the studied patients

\begin{tabular}{|l|c|}
\hline Cut off point & $>25.8$ \\
\hline Accuracy & $96.60 \%$ \\
\hline P-value & $<0.001^{* *}$ \\
\hline Sensitivity & $96.70 \%$ \\
\hline Specificity & $75.00 \%$ \\
\hline 95\% CI & $0.929-1$ \\
\hline
\end{tabular}

**: Highly significant

\section{DISCUSSION}

Our study found that there was a statistically highly significant positive linear correlation between insulin level and severity of coronary artery disease as assessed by Gensini score in type 2 diabetic patients. This agreed with Mahabala et al. ${ }^{(9)}$ who revealed strong correlation between insulin resistance and severity of coronary stenosis assessed by Gensini score and their results depended upon accelerated atherosclerosis as a major burden of diabetes and the risk of advanced atherosclerosis was fivefold higher in type 2 diabetic subjects than in nondiabetic individuals. Also, Hamza et al. ${ }^{(10)}$ found that in diabetic NSTEMI patients, coronary artery disease was more severe and located in distality with a bad downstream quality, coronary lesions were more extensive, with higher rate of tritroncular lesions. Lesions were significantly longer, the link between the pathogeneses of diabetes and atherosclerosis is explained by insulin resistance phenomenon, which causes dyslipidemia and hyperglycemia, leading to reduced nitric oxide synthesis in artery walls ${ }^{(\mathbf{1 0})}$.

Similarly, Uppunda et al. ${ }^{(11)}$ showed that an increase in insulin resistance increases the risk of severity of CAD in patients with metabolic syndrome, SYNTAX score was used to measure severity of CAD. In harmony to our results Tsuchihashi et al. ${ }^{(12)}$, found that hyperinsulinemia is a risk for CAD because diabetes mellitus, hypertension, and hyperlipidemia are known to aggregate and to have synergistic effects on the occurrence and the progression of CAD. Insulin resistance is known to have a link to hypertension, hypertriglyceridemia, and decreased high density lipoprotein cholesterol, and is considered to play a key role in multi-risk factor syndromes like metabolic syndrome, insulin resistance syndrome.

In concordance with Granér $\boldsymbol{e t}$ al. ${ }^{(13)}$, who showed that patients with more severe degree of insulin resistance have a more severe, extensive, and distal type of coronary artery disease than patients with lower degree of insulin resistance, as insulin resistance has been associated with obesity, predominantly intraabdominal distribution of fat, hypertension, low highdensity lipoprotein (HDL) cholesterol, and hypertriglyceridemia, all of which are well known risk factors for CAD. This was concordant with Eddy $\boldsymbol{e}$ t al. (14), who reported that insulin resistance was the single most frequent cause of coronary artery disease. Their study estimated the proportion of coronary artery disease due to insulin resistance and found that insulin resistance is responsible for approximately $42 \%$ of myocardial infarctions. This effect on coronaries is indirect and mediated through its effects on other variables such as systolic blood pressure (SBP), HDL cholesterol, triglycerides, glucose, and apoB. Each of those variables, in turn, is affected by other variables such as age, sex, and race. Also, the role of obesity which is the major cause of insulin resistance in the etiology of both diabetes and CAD ${ }^{(14)}$.

On the contrary, Adler et al. ${ }^{(15)}$, found no association between HOMA-IR and coronary disease and among patients with newly diagnosed type 2 diabetes, insulin resistance was not a risk factor for cardiovascular disease. This is because the selected studied population were shortly after diagnosis of diabetes and the effect of insulin resistance were modified by duration of diabetes.

The present study showed statically highly significant negative linear correlation between ejection fraction and insulin resistance. This agreed with Cauwenberghs et al. (16), who found that increased insulin resistance deteriorated the left ventricular systolic function and it depended upon predicted LV hypertrophy and worsening in LV systolic (EF\%) and diastolic function over time.

Similarly, Ehl et al. ${ }^{(17)}$, found that LVEF was significantly lower in diabetic patients treated with insulin compared with non-diabetic patients or those not treated with insulin in a large patient population, as insulin has profound effects on the myocardium by affecting the energy metabolism, sympathetic activity, inflammation, oxidative stress, and microvascular dysfunction. MacDonald et al. ${ }^{(18)}$, reported that left 
ventricular systolic dysfunction and diabetes mellitus commonly coexist. Each condition increases the likelihood of developing the other and insulin resistance occurs in HF of both ischemic and nonischemic etiology and many mechanisms have been suggested including sympathetic nervous system overactivity, sedentary lifestyle, endothelial dysfunction, loss of skeletal muscle mass, and influence of cytokines such as TNFalpha and leptin on peripheral insulin sensitivity. On the other side diabetes develops left ventricular systolic dysfunction and heart failure as a result of risk factors for $\mathrm{HF}$ which are common in diabetics, like hypertension, may have a direct effect on the myocardium and also DM may activate neurohormonal systems ${ }^{(18)}$.

In our study we found that $\mathrm{EF} \%$ was significantly lower in patients with severe coronary artery disease compared to other groups and that was concordant with Jia et al. ${ }^{(19)}$ and Chatterjee and Rame (20), who showed the severity of coronary atherosclerosis was a strong and statistically highly significant predictor of LVEF level and dysfunction of LV ejection independent of the other major risk factors (age, BMI, SBP, FBG, blood lipid count and leucocyte count) the possible mechanism for systolic heart failure is ischemic heart disease, and adverse remodeling is associated with an unfavorable prognosis. In patients with acute coronary syndromes, even after successful recanalization of the infarct-related artery, the risk of development of heart failure and mortality increases with increasing end-diastolic volume and end-systolic volume, and is inversely related to the ejection fraction. Naguib et al. ${ }^{(21)}$ found a strong negative correlation between Gensini score EF \% and this goes with the fact that increased number of affected vessels impairs perfusion of larger myocardial area and hence impair function and the higher Gensini score is associated with more severe coronary artery disease, the lower LV function.

In this study insulin level was higher in hypertensive patients and this went in harmony with Natali et al. (22), who concluded that the acute antinatriuretic effect of insulin is the mechanism through which insulin can cause and contribute to the development of essential hypertension. Other possible explanation may be that insulin proportionally increased plasma norepinephrine levels and caused sympathetic nervous system activation that affected blood pressure (23, 24). Moreover, we found that hypertension was more prevalent in patients with $\mathrm{CAD}$ than those with normal coronary and that was concordant with Nakanishi et al. ${ }^{(25)}$, who found that hypertensive patients had greater extent of coronary atherosclerosis and greater risk of major adverse cardiac events compared with non-hypertensive patients. The principal underlying pathophysiologic mechanism is considered to be mechanically related to pulse pressure as wide pulse pressure has been reported to be associated with increased cardiac events. Both increased pulse pressure and systolic pressure contribute to endothelial dysfunction, which facilitates the entry of low-density lipid cholesterol into the blood vessel wall, initiating the atherosclerotic process. Hypertension also is a cause of left ventricular hypertrophy, which has been implicated as a cause of coronary atherosclerosis ${ }^{(25)}$.

Our study showed significant relation between insulin resistance and dyslipidemia and that agreed with Goldberg et al. ${ }^{(26)}$, who showed that insulin resistance caused diabetic dyslipidemia as result of liver production of apolipoprotein $\mathrm{B}$, the major protein component of very low-density lipoprotein (VLDL) and LDL, is increased in type 2 diabetes and also defects in insulin action and hyperglycemia could lead to changes in plasma lipoproteins in patients with diabetes. Moreover, Zheng et al. ${ }^{(27)}$, found that normal glucose tolerance individuals with dyslipidemia had severe insulin resistance as result of impaired pancreatic $\beta$ cells function caused by dyslipidemia.

Our study revealed that total cholesterol, low density lipoprotein (LDL) and triglycerides (TG) were significantly higher in patients with higher Gensini scores than those with lower scores. On the contrary HDL was significantly lower in patients with higher Gensini scores than those with lower scores Similarly, Tarchalski et al. ${ }^{(28)}$, found that the extent of coronary atherosclerosis is positively correlated with atherogenic lipids, i.e. total cholesterol, LDL cholesterol and TG, which can enter cells via apo B/E receptors or through an unregulated scavenger receptor that present in smooth muscle cells and macrophages, lead to accumulation of intracellular cholesterol and formation of foam cells in which oxidation occurs. Foam cells can rupture leading to the release of oxidized lipoproteins, proteolytic enzymes and toxic oxygen derivatives that can altogether damage the vessel wall and promote atherosclerotic changes within arteries It is also negatively correlated with antiatherogenic HDL cholesterol which explained by antiatherogenic properties of HDL particles as HDL is engaged in reverse cholesterol transport from cells and atherosclerotic plaques into the liver or to other tissues (28)

Moreover, Jenkins et al. ${ }^{(29)}$, found that the concentration of high-density lipoprotein (HDL) showed a strong inverse association with severity of $\mathrm{CAD}$, which was independent of the effects of age and other lipoproteins. On the other hand, total plasma cholesterol, low-density lipoprotein (LDL) cholesterol, and the combined effect of LDL cholesterol plus verylow-density lipoprotein triglyceride showed direct, significant correlations with $\mathrm{CAD}$, but these were weaker than that of HDL.

In our study $\mathrm{HgA} 1 \mathrm{C}$ was significantly higher in patients with higher Gensini scores than those with lower scores. Similarly, Gaber et al. ${ }^{(30)}$, showed that there was a statistically significant positive correlation between HbA1c levels and Gensini scores, as diabetes 
mellitus is known to cause microvascular and possibly macrovascular complications as it is one of the major risk factors for CADs. Observations from many clinical trials showed that DM is associated with a higher prevalence of CAD with higher morbidity and more fatal coronary events due to a higher incidence of plaque rupture and superimposed thrombosis in diffusely diseased coronary arteries.

\section{CONCLUSION}

Fasting insulin level is a simple test for insulin resistance and significantly correlated with severe CAD so it may have a value as a predictor of severity of CAD and risk stratification of diabetic patients.

\section{REFERENCES}

1. Lozano R, Naghavi M, Foreman $K$ et al. (2012): Global and regional mortality from 235 causes of death for 20 age groups in 1990 and 2010: a systematic analysis for the Global Burden of Disease Study 2010. The Lancet, 380(9859):2095128.

2. Arad Y, Newstein D, Cadet F et al. (2001): Association of multiple risk factors and insulin resistance with increased prevalence of asymptomatic coronary artery disease by an electron-beam computed tomographic study. Arterioscler Thromb Vasc Biol., 21:2051-2058

3. Hegazi R, El-Gamal M, Abdel-Hady N (2015): Epidemiology of and risk factors for type 2 diabetes in Egypt. Annals of Global Health, 81: 814-820.

4. Reaven G (1988): Role of insulin resistance in human disease. Diabetes, 37:1595-607.

5. Kendall D, Cuddihy R, Bergenstal $R$ (2009): Clinical application of incretin-based therapy: Therapeutic potential, patient selection and clinical use. Am J Med., 122: S37-50.

6. Matthews D, Cull C, Stratton I et al. (1998): UKPDS 26: Sulphonylurea failure in non-insulin-dependent diabetic patients over six years. UK prospective diabetes study (UKPDS) group. Diabet Med., 15:297-303.

7. Neeland I, Patel R, Eshtehardi P et al. (2012): Coronary angiographic scoring systems: an evaluation of their equivalence and validity. American Heart Journal, 164(4):547-52.

8. Gensini G (1983): A more meaningful scoring system for determining the severity of coronary heart disease. Am J Cardiol., 51:606-611.

9. Mahabala C, Srinivasan M, Manjrekar P et al. (2013): Correlation of severity of coronary artery disease with insulin resistance. North American Journal of Medical Sciences, 10: 611-616.

10. Hamza S, Ali H, Najla K et al. (2018): Impact of type 2 diabetes in non ST-elevation myocardial infarction (NSTEMI). International Journal of Advanced Research, 6(12): 1029-1033.

11. Uppunda D, Shetty R, Rao $P$ et al. (2019): Insulin resistance and the severity of coronary artery disease based on obesity status in stable-angina patients: A cross-sectional analysis. Journal of Applied Pharmaceutical Science, 9 (7): 91-94.

12. Tsuchihashi K, Hikita N, Hase $M$ et al. (1999): Role of hyperinsulinemia in atherosclerotic coronary arterial disease: studies of semiquantitative coronary angiography. Intern Med., 38:691-697.
13. Granér M, Syvänne M, Kahri J et al. (2007): Insulin resistance as predictor of the angiographic severity and extent of coronary artery disease. Annals of Medicine, 39(2): 137142.

14. Eddy D, Schlessinger L, Kahn R et al. (2009): Relationship of insulin resistance and related metabolic variables to coronary artery disease: a mathematical analysis. Diabetes Care, 32(2):361-6.

15. Adler A, Levy J, Matthews D et al. (2005): Insulin sensitivity at diagnosis of Type 2 diabetes is not associated with subsequent cardiovascular disease (UKPDS 67). Diabetic Medicine, 3:306-311.

16. Cauwenberghs N, Knez J, Thijs L et al. (2018): Relation of insulin resistance to longitudinal changes in left ventricular structure and function in a general population. Journal of the American Heart Association, 7(7): 8315-8321.

17. Ehl N, Ku hne M, Brinkert M et al. (2011): Diabetes reduces left ventricular ejection fraction-irrespective of presence and extent of coronary artery disease, European Journal of Endocrinology, 165: 945-951.

18. MacDonald M, Petrie M, Hawkins $N$ et al. (2008): Diabetes, left ventricular systolic dysfunction, and chronic heart failure. European Heart Journal, 29(10):1224-40.

19. Jia E, Xu Z, Yang Z et al. (2011): Severity of coronary atherosclerosis is an independent predictor of the left ventricular ejection fraction. Clinical and Experimental Pharmacology and Physiology, 2:109-12.

20. Chatterjee K, Rame J (2008): Systolic heart failure: chronic and acute syndromes. Critical Care Medicine, 36(1): 44-51.

21. Naguib T, Elsherbeiny E, Elzayyat A (2014): Predictors of depressed left ventricular function in patients presenting with ST-elevation myocardial infarction. ZUMJ., 6:786-798.

22. Natali A, Quiñones Galvan A, Santoro D et al. (1993): Relationship between insulin release, antinatriuresis and hypokalaemia after glucose ingestion in normal and hypertensive man. Clinical Science, 3:327-35.

23. Berne C, Fagius J, Niklasson F (1989): Sympathetic response to oral carbohydrate administration. Evidence from microelectrode nerve recordings. The Journal of Clinical Investigation, 84(5):1403-9.

24. Rooney D, Edgar J, Sheridan B et al. (1991): The effects of low dose insulin infusions on the renin angiotensin and sympathetic nervous systems in normal man. European Journal of Clinical Investigation, 4:430-435.

25. Nakanishi R, Baskaran L, Gransar H et al. (2017): Relationship of hypertension to coronary atherosclerosis and cardiac events in patients with coronary computed tomographic angiography. Hypertension, 70(2):293-9.

26. Goldberg I (2001): Diabetic dyslipidemia: causes and consequences. The Journal of Clinical Endocrinology and Metabolism, 86(3):965-71.

27. Zheng S, Xu H, Zhou H et al. (2017): Associations of lipid profiles with insulin resistance and $\beta$ cell function in adults with normal glucose tolerance and different categories of impaired glucose regulation. PLoS One, 12(2): 172221.

28. Tarchalski J, Guzik P, Wysocki H (2003): Correlation between the extent of coronary atherosclerosis and lipid profile. Mol Cell Biochem., 246(1-2):25-30.

29. Jenkins P, Harper R, Nestel P (1978): Severity of coronary atherosclerosis related to lipoprotein concentration. Br Med J., 2(6134):388-91.

Gaber A, El-Kholy T, Aziz E et al. (2014): Glycosylated haemoglubin level and severity of Coronary Artery Disease. Med J Cairo Univ., 82: 87-93. 